\title{
Defect Features Recognition in 3D Industrial CT Images
}

\author{
Haina Jiang \\ Chongqing College of Electronic Engineering, Chongqing City, 401331, China \\ Corr. address: No. 70-2-4-3, Huxi Garden, University Town, Shapingba District, Chongqing City, 401331, China \\ E-mail: hainjiangcq@126.com
}

\section{Technical Paper}

Keywords: industrial CT image, defect location, image segmentation, feature extraction, feature recognition

Received: September 15, 2018

\begin{abstract}
Due to the limitations of production conditions, there is a certain probability that workpiece product has internal defects, which will have a certain impact on the performance of workpiece. Therefore, the internal defects detection of workpiece is essential. This study proposed a defect recognition method based on industrial computed tomography $(C T)$ image to identify the internal defects of workpiece. The block fractal algorithm was used to locate the defect parts of the image, then the improved k-means clustering algorithm was used to segment the defect parts, and feature vector was extracted by $\mathrm{Hu}$ invariant moments. Finally, the firefly algorithm and radial basis function (RBF) neural network were combined to identify the defect. It was found from the experiments that the algorithm in this study had the accuracy of $97.89 \%$, which proved the reliability of the algorithm and provided some suggestions for the defects recognition.
\end{abstract}

Povzetek: Za prepoznavanje okvar na 3D slikah industrijskih izdelkov je uporabljena metoda vinske mušice.

\section{Introduction}

Defect detection plays a very important role in the industrial field. Through defect detection, product quality can be effectively improved. Alimohamadi et al. [1] proposed a new defect detection method based on the optimal Gabor wavelet filters, which combined with morphological analysis. The experimental results on different type of textiles showed that this algorithm was robust for defects detection of various kind of textile. Chen et al. [2] proposed a new defect detection method based on dual-tree complex wavelet transform (DT-CWT) and took advantage of near shift-invariance of DT-CWT to extract weak defect feature. Experimental results demonstrated the validity of the proposed method. Sabeenian et al. [3] presented an algorithm of defect detection by making use of multi-resolution combined statistics and spatial frequency method. The accuracy obtained by the simulation using MATLAB was found to be $99 \%$, which proved the practicality of the algorithm. Liu et al. [4] optimized subtractive clustering method (SCM) by Akaike information criterion (AIC) and then constructed radial basis function (RBF) model by using the obtained AIC-SCM algorithm, which improved the adaptability of the RBF model. Experimental results showed that this method could identify defects with a high accuracy. Leng et al. [5] used convolutional neural network method in the detection and classification of galvanized stamping parts and obtained a precision of $99.6 \%$. Industrial computed tomography (CT) image is a simple and efficient method [6,7] for internal defect detection of workpiece, which can effectively detect internal defects and significantly reduce the detection cost [8]. Samarawickrama et al. [9] made defect detection of tile based on industrial CT images and found it was more accurate and efficient than the manual method. In this study, based on the industrial CT image, the defect was obtained through the localization and segmentation of the defect image, then the feature extraction was conducted by using $\mathrm{Hu}$ invariant moments, and finally the RBF neural network which was optimized by using the firefly algorithm was used for recognizing defects to explore the reliability of this method in defect recognition.

\section{Internal defect detection of workpiece}

Due to the production technology, production conditions and other aspects, workpiece products often have a certain probability of internal defects. These defects not only affect the performance of workpiece, but also have certain safety risks in the actual use process. Therefore, the detection of defects is an important part of the industrial production. Industrial CT image is an effective method for workpiece defect detection. With the development of technology, the performance of industrial CT image is gradually improving, and its cost is also reducing. It has been widely used in aerospace, military, electronics, petroleum and other fields. Industrial CT images can easily be stored and analyzed, and enable quick and accurate detection of the presence or absence of defects in workpiece, as well as evaluation of the size and location of defects [10-12]. It has higher resolution 


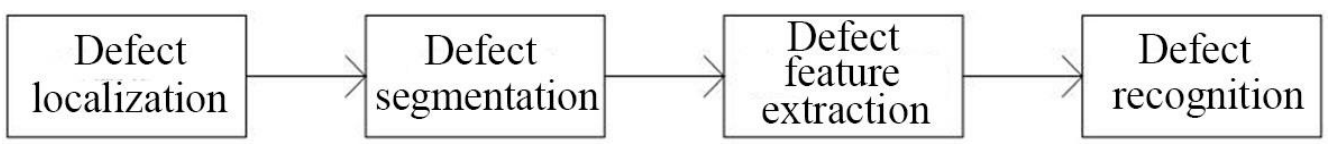

Figure 1: Flow chart of industrial CT image defect recognition.

and adaptability, so images of different gray levels can be effectively detected. At present, the detection of defects in CT images is mostly carried out manually with low accuracy. An intelligent identification method can effectively improve efficiency and reduce errors. This is the development direction of defect detection methods.

The process of defect detection based on industrial CT images proposed in this study is shown in Figure 1.

\section{Defect detection algorithm}

\subsection{Defect localization algorithm based on block fractal}

Automatic defect localization was performed using fractal theory [13]. Fractal theory is put forward by Mandelbrot, which has been extensively applied in graphics and geography. Fractal dimension [14] was obtained by Blanket algorithm which is put forward by Peleg; then the block with defects was marked, and the defect was localized.

\subsubsection{Blanket algorithm}

Suppose the gray function of the image is $h(i, j)$, imagine a blanket covering the gray surface of the image, suppose the upper surface as $O_{\delta}(i, j)$, the lower surface as $U_{\delta}(i, j)$, and scale as $\{\delta \mid \delta=1,2, \cdots, N\}$, then the upper and lower surface under different scales can be expressed as:

$$
\begin{gathered}
O_{0}(i, j)=U_{0}(i, j)=h(i, j),(1) \\
O_{\delta}(i, j)=\max \left\{O_{\delta-1}(i, j)+1, \max _{|(m, n)-(i, j)| \leq 1} O_{\delta-1}(m, n)\right\},
\end{gathered}
$$

$$
U_{\delta}(i, j)=\min \left\{U_{\delta-1}(i, j)+1, \min _{|(m, n)-(i, j)| \leq 1} U_{\delta-1}(m, n)\right\} .
$$

According to the above expressions, the area and volume of gray surface can be calculated, and the fractal area $A_{\delta}$ can be obtained. The relation between the fractal area and the fractal dimension is:

$$
A_{\delta} \approx \beta \delta^{2-W},
$$

where $\mathrm{W}$ stands for the fractal dimension, and $\beta$ stands for the constant. The following expression can be obtained by calculation:

$$
\log A_{\delta} \approx(2-W) \log \delta+\log \beta \text {. (5) }
$$

We see that the area of the fractal dimension is linearly related to the logarithm of the fractal dimension, and the slope of the line can be calculated to obtain the fractal dimension $W$ :

$$
W=2-\frac{N \sum_{i} \log \delta_{i} \log A_{\delta_{i}}-\sum_{i} \log \delta_{i} \sum \log A \delta_{i}}{N \sum_{i}\left(\log \delta_{i}\right)^{2}-\left(\sum_{i} \log \delta_{i}\right)^{2}}
$$

\subsubsection{Block fractal algorithm}

(1) The image is divided into rectangular regions of the same size.

(2) The corresponding fractal area $A \delta_{i}$ of different scales $\delta_{i}$ on each region is calculated by the Blanket algorithm, and $\left(A_{\delta_{i}}, \delta_{i}\right)$ can be obtained.

(3) The fractal dimension $\mathrm{W}$ can be calculated according to formula (6).

(4) Mark the fractal threshold as K. If the fractal dimension of the block is greater than this threshold, it indicates that there is an edge, and the part with the edge is marked with white.

(5) Determine if the marked blocks are workpiece edges or defect areas. As the number of blocks in defect areas is less than the workpiece edges, through calculation, if the number of blocks is greater than the connected threshold value $\mathrm{T}$, it means that they are workpiece edges. (6) Remember the defect area of the workpiece after removing the workpiece edge.

\subsection{Image segmentation algorithm based on improved k-means clustering}

The traditional K-means clustering algorithm may reduce the reliability. To make up the deficiencies of the algorithm, the initial clustering center automatic generation algorithm [15] was used to improve the traditional algorithm.

Suppose that $\mathrm{T}$ is the coordinate set of image data set $\mathrm{D}, h(x, y, z)$ stands for the gray value of somewhere in $\mathrm{D}$, $V_{1}^{i}, \cdots, V_{k}^{i}$ stands for $\mathrm{K}$ classes in the clustering process, $C_{1}^{i}, \cdots C_{k}^{i}$ stands for $\mathrm{K}$ clustering centers, and i stands for the number of iterations. The algorithm steps are as follows:

(1) Determine $\mathrm{K}$ and the accuracy of clustering $\delta$.

(2) The clustering center is generated by the initial clustering center automatic generation algorithm.

(3) Take each initial clustering center as the set member of the initial cluster $V_{1}^{i}, \cdots, V_{k}^{i}$, and $C_{1}^{1} \in V_{1}^{1}, \cdots, C_{k}^{1} \in V_{k}^{1}$.

(4) Conduct the iterations and divide $h(x, y, z)$ into one cluster according to the minimum distance, i.e.,

$$
\begin{aligned}
& D\left(T, C_{l}^{i}\right)<D\left(T, C_{j}^{i}\right), \text { then } h(x, y, z) \in V_{l}^{i}, \\
& D\left(T, C_{l}^{i}\right) \geq D\left(T, C_{j}^{i}\right), \text { then } h(x, y, z) \in V_{j}^{i},
\end{aligned}
$$

where $j=1, \cdots, k ; l=1, \cdots, k ; j \neq l, D\left(T, C_{l}^{i}\right)$ stands for the distance function of the algorithm, i.e., the distance between $h(x, y, z)$ and the clustering center in the $\mathrm{i}$-th iteration.

(5) Reset the clustering center and then cluster again. Suppose $h(x, y, z) \in V_{l}^{i}$, then its clustering center is: 


$$
Z_{l}^{i+1}=\frac{1}{N_{l}} \sum h(x, y, z),
$$

where $N_{l}$ stands for the feature points number of $C_{l}^{i}$ at the i-th iteration. The resetting of clustering center of $h(x, y, z) \in V_{j}^{i}$ is also carried out.

(6) Repeat (4) and (5) until the clustering center remains unchanged $Z^{i+1}=Z^{i}$ or $\left|Z^{i+1}-Z^{i}\right| \leq \delta$.

(7) Output image segmentation results according to clustering results. The number of clustering $\mathrm{K}$ stands for the number of peak values of gray histogram of reference image or the type of reference image object. The distance function is:

$$
D\left(T, C_{l}^{i}\right)=\sqrt{\left(h(x, y, z)-C_{l}^{i}\right)^{2}} .
$$

\subsection{Feature extraction algorithm based on Hu invariant moments}

Three common defects in workpieces are stomata, cracks and slag inclusion. The shape and gray information of the three defects are very different, and the feature information can be extracted for identification.

(1) Shape features

(1) The length-width ratio of the defect part is $z=\frac{R}{K}$, where $\mathrm{R}$ stands for the long axis and $\mathrm{K}$ stands for the short axis.

(2) The circularity of the defect is $e=\frac{L^{2}}{A}$, where $\mathrm{L}^{2}$ stands for square of circumference and A stands for area. (2) Gray information

$$
\text { mean }=\frac{\sum_{x=R_{\min }}^{R_{a m x}} \sum_{y=L_{\min }}^{L_{\max }} h(x, y)}{n},
$$

Establishment of RBF neural network

The number of nodes in the input layer and output layer of the neural network is 14 and 3, the hidden layer and input layer are the same, and 001, 010 and 100 stands for the stomata, cracks and slag inclusion respectively.

(1) Weight threshold optimization

(1) Initialize parameters: $\rho$ stands for the volatilization rate of luciferin at $t-1, \gamma$ stands for the update rate of luciferin, $\beta$ stands for the change rate of field, $s$ stands

$$
v=\frac{\sum_{x=R_{\min }}^{R_{\max }} \sum_{y=L_{\min }}^{L_{\max }}(h(x, y)-\text { mean })^{2}}{n},
$$

where Mean stands for the Mean value of grayscale image, $v$ stands for the variance of grayscale, $h(x, y)$ stands for the gray value of the defect pixel points, and $n$ stands for the number of pixels.

Seven invariant moments, $\varphi_{1}-\varphi_{7}$, can be obtained according to $\mathrm{Hu}$ invariant moment theory [16]. Table 1 is obtained after abstraction on the moments.

\begin{tabular}{|l|l|l|l|l|}
\hline $\mathrm{R} 1$ & $\mathrm{R} 2$ & $\mathrm{R} 3$ & $\mathrm{R} 4$ & $\mathrm{R} 5$ \\
\cline { 1 - 3 }$\frac{\sqrt{\varphi_{2}}}{\varphi_{1}}$ & $\frac{\varphi_{1}+\sqrt{\varphi_{2}}}{\varphi_{1}-\sqrt{\varphi_{2}}}$ & $\frac{\sqrt{\varphi_{3}}}{\varphi_{4}}$ & $\frac{\sqrt{\varphi_{3}}}{\sqrt{\left|\varphi_{5}\right|}}$ & $\frac{\sqrt{\varphi_{4}}}{\sqrt{\left|\varphi_{5}\right|}}$ \\
\hline R6 & $\mathrm{R} 7$ & $\mathrm{R} 8$ & $\mathrm{R} 9$ & $\mathrm{R} 10$ \\
\hline$\frac{\left|\varphi_{6}\right|}{\varphi_{1} \times \varphi_{3}}$ & $\frac{\left|\varphi_{6}\right|}{\varphi_{1} \times \sqrt{\left|\varphi_{5}\right|}}$ & $\frac{\left|\varphi_{6}\right|}{\varphi_{3} \times \sqrt{\left|\varphi_{2}\right|}}$ & $\frac{\left|\varphi_{6}\right|}{\sqrt{\varphi_{2} \times\left|\varphi_{5}\right|}}$ & $\frac{\left|\varphi_{5}\right|}{\varphi_{3} \times \varphi_{4}}$ \\
\hline
\end{tabular}

Table 1: Feature value obtained from the abstracted $\mathrm{Hu}$ invariant moment.

The above 10 feature values, two shape features (length-width ratio and degree of circularity) and two gray features (gray average and gray variance) can be used as feature vectors to identify the defects.

\subsection{Defect recognition algorithm based on firefly neural network}

In this study, a combination of firefly algorithm and RBF neural network was adopted to identify the defect feature. The flowchart of the algorithm is shown in Figure 2.

for the moving step length, $r s$ stands for the threshold of the perceived range of firefly, and $n t$ stands for the threshold of the number of neighbor fireflies.

(2) Initialization algorithm: The current position of firefly $\mathrm{i}$ is $X_{i}(t), i=1,2,3, \cdots, N$, each firefly has the same luciferin $l_{0}$ and the same decision radius $n$.

(3) The update formula of luciferin is $l_{i}(t)=(1-\rho) l_{i}(t-1)+\not f f\left(X_{i}(t)\right), l_{i}(t-1)$ stands for the luciferin of firefly $i$ at $t-1, f\left(X_{i}(t)\right)$ stands for the

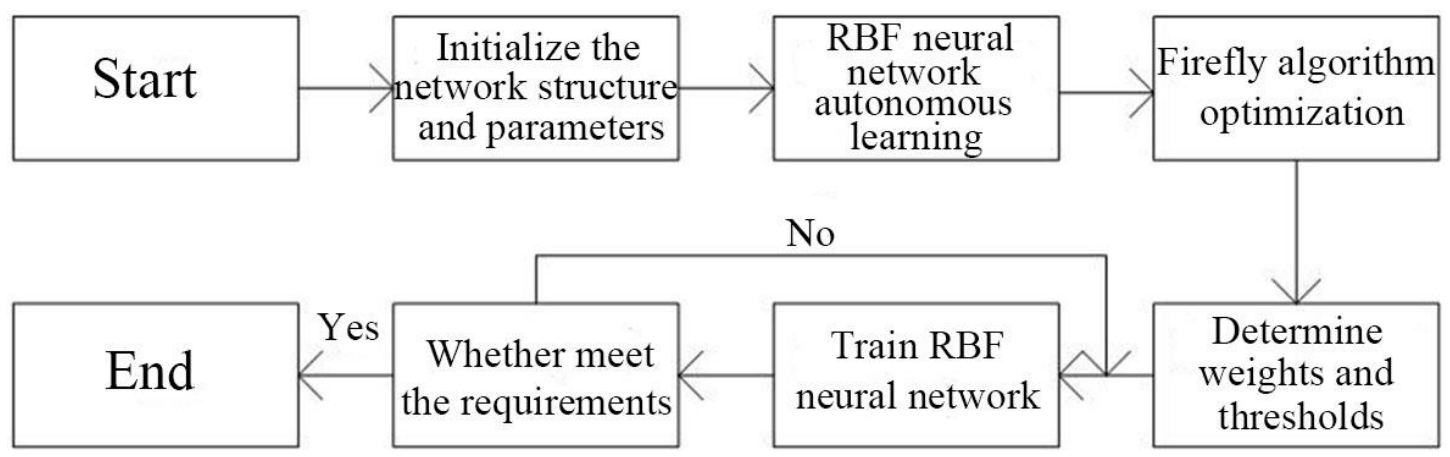

Figure 2: Flow chart of firefly neural network. 
position fitness value of firefly $i$ at $t$.

(4) The neighbor fireflies set formula is $N_{i}(t)=\left\{j:\left\|X_{j}(t)-X_{i}(t)\right\|<r_{i}(t) ; l_{i}(t)<l_{j}(t)\right\} \quad, \quad$ where $N_{i}(t)$ stands for the neighbor fireflies set of fireflies $i$ at $\mathrm{t},\|\bullet\|$ stands for the Euclidean distance, and $r_{i}(t)$ stands for the decision radius of fireflies $i$ at $t$. The probability that firefly $i$ chooses $\mathrm{j}$ as neighbor firefly is $P_{i j}(t)=\frac{l_{j}(t)-l_{i}(t)}{\sum_{k \in N_{i}(t)} l_{k}(t)-l_{i}(t)}$, the position updating formula is $X_{i}(t+1)=X_{i}(t)+s\left(\frac{X_{j}(t)-X i(t)}{\left\|X_{j}(t)-X i(t)\right\|}\right.$, and the updating formula of decision radius is $r_{i}(t)=\min \left\{r s, \max \left[0, r_{i}(t)+\beta\left(n t-\left|N_{i}(t)\right|\right]\right\}\right.$, where $r_{i}(t)$ stands for the perceived range of firefly $\mathrm{i}$ at $\mathrm{t}$, $0<r_{i}(t)<r s$, and $\left|N_{i}(t)\right|$ stands for the size of neighbor set.

(5) After the iteration, it is judged whether the iteration number reaches the maximum. If it does, the algorithm is finished and the optimal value is recorded; if not, the iteration is continued.

(3) The above data are used as training samples for neural network testing and training.

\section{Example analysis of defect workpiece}

In order to verify the correctness of the method in this study, defect recognition was carried out on 100 industrial CT images of solid rocket engine model which was in a size of $512 \times 512$. The material of the motor body was $30 \mathrm{GrMnSiA}$, the length of the motor grain was $1000 \mathrm{~mm}$, and the external diameter was 150 $\mathrm{mm}$. The artificial detection results were 80 defective images, and 20 non-defective images and 142 defects).

\subsection{Defect localization results}

The defects of the industrial CT images were positioned.

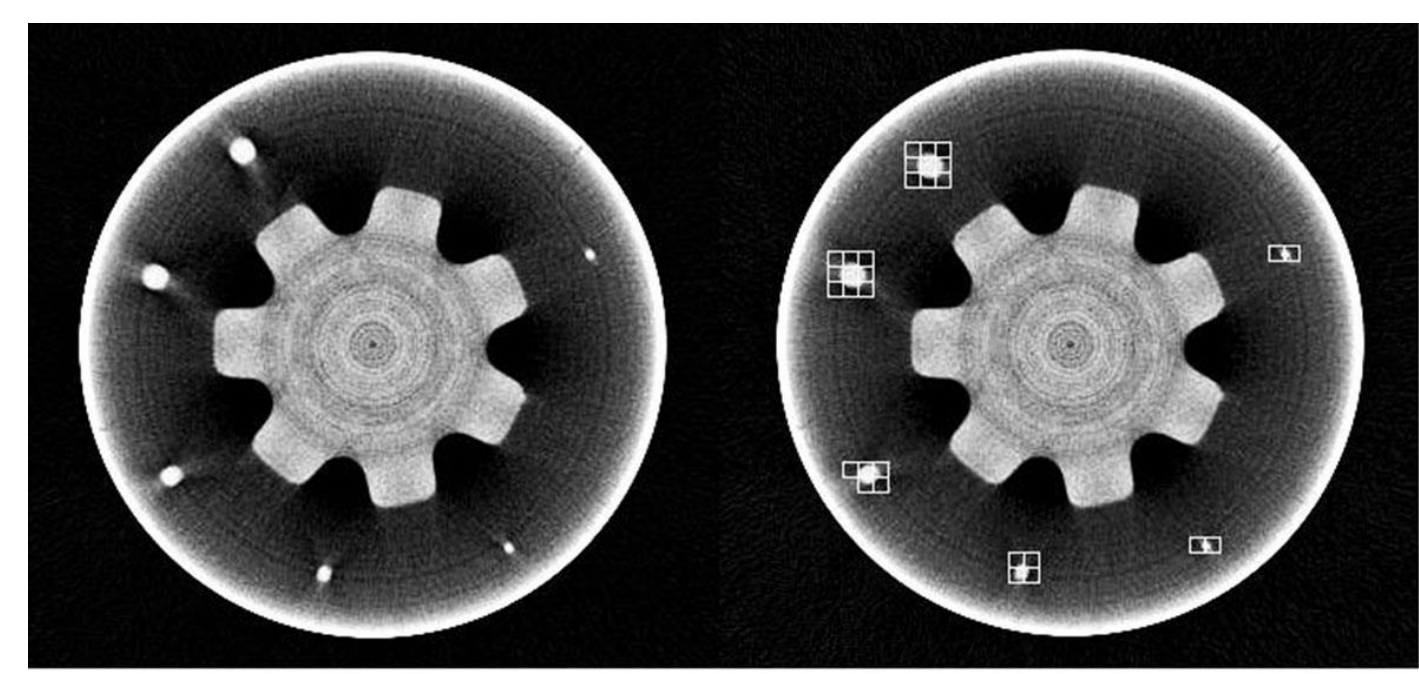

Industrial CT image

Figure 4: Defect localization results.
The size of block was a quarter. The calculation results of fractal dimension are shown in Figure 3.

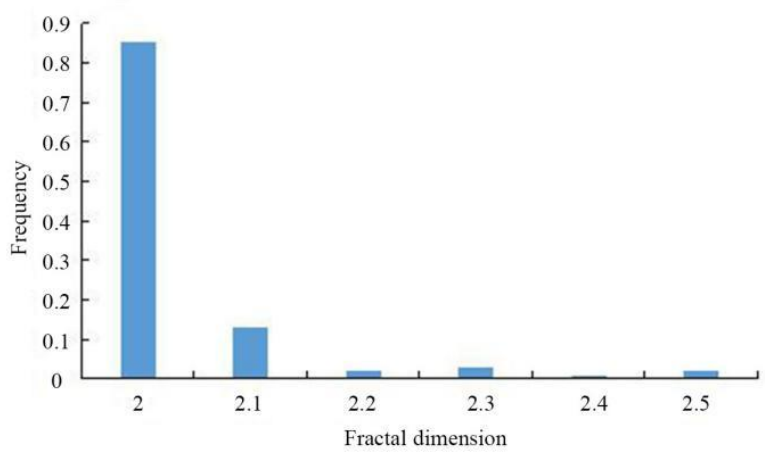

Figure 3: The frequency distribution of fractal dimension.

Fractal dimension has a large influence on the accuracy of defect marks, it can be noted from Figure 3 that image defects could be clearly positioned when the fractal dimension was between 2 and 2.1 and the frequency was 0.85 . Therefore, the fractal dimension was set as 2.1. The defect localization results are shown in Figure 4.

As shown in Figure 3, the algorithm used in this study can locate the defect area of the workpiece accurately and facilitate the subsequent defect detection.

\subsection{Defect segmentation results}

One hundred industrial CT images (80 defective images and 20 non-defective images) were processed by the improved k-mean clustering algorithm, and the results obtained were compared with the results of manual judgment, as shown in Table 2.

As can be seen from the Table 2, the algorithm successfully segmented 78 defective images, only one non-defective image was wrongly segmented, and the overall segmentation accuracy rate was relatively high, indicating that the proposed segmentation algorithm was highly reliable. 


\begin{tabular}{|l|l|l|}
\hline & $\begin{array}{l}\text { Defective } \\
\text { images }\end{array}$ & $\begin{array}{l}\text { Non-defective } \\
\text { images }\end{array}$ \\
\hline $\begin{array}{l}\text { Number of segmented } \\
\text { defects }\end{array}$ & 78 & 1 \\
\hline $\begin{array}{l}\text { Number of } \\
\text { unsegmented defects }\end{array}$ & 2 & 19 \\
\hline Accuracy rate & $97.5 \%$ & $95 \%$ \\
\hline
\end{tabular}

Table 2: Defect segmentation results.

\subsection{Feature extraction results}

The feature extraction of defect images was carried out by the method of abstract invariant moments. Taking the stomata as an example, its feature quantity is shown in Table 3.

\begin{tabular}{|l|l|l|l|}
\hline & $\begin{array}{l}\text { Original } \\
\text { image }\end{array}$ & $\begin{array}{l}\text { Translated } \\
\text { Image }\end{array}$ & $\begin{array}{l}\text { Image which is } \\
\text { clockwise rotated } \\
\text { for 90 degrees }\end{array}$ \\
\hline R1 & 0.315687 & 0.314256 & 0.312456 \\
\hline R2 & 1.935621 & 1.935124 & 1.935214 \\
\hline R3 & 4.500254 & 4.502103 & 4.505321 \\
\hline R4 & 3.785214 & 3.782158 & 3.780215 \\
\hline R5 & 2.124521 & 2.125632 & 2.120325 \\
\hline R6 & 0.234665 & 0.239654 & 0.236589 \\
\hline R7 & 0.621453 & 0.621036 & 0.625879 \\
\hline R8 & 0.235462 & 0.231456 & 0.236587 \\
\hline R9 & 0.625471 & 0.620852 & 0.623168 \\
\hline R10 & 0.442123 & 0.441258 & 0.446852 \\
\hline
\end{tabular}

Table 3: Defect feature value of stomata.

These 10 feature values were extracted, plus two shape features and two gray features, a total of 14 feature vectors were obtained.

\subsection{Feature recognition results}

The theoretical output value and actual output value of 10 defects identified in 8 pictures are shown in Table 4.

The theoretical output values of the neural network should be 001 (crack), 010 (stomata) and 100 (slag inclusion), but there always exists error in the actual output. Therefore, the error was controlled to 0.2 , and the actual output less than 0.2 was rounded to 0 , while larger than 0.8 was rounded to 1 . Only the recognition of A7 was wrong in the ten defects of the Table 4. The 142 defects in the processed $100 \mathrm{CT}$ images were recognized, and 139 defects were correctly recognized, and 3 defects were misjudged. The accuracy rate was $97.89 \%$, which indicated that the defect recognition method in this study had high reliability.

\section{Discussion and conclusion}

The internal defects of a workpiece can greatly affect the practicability and safety of the workpiece. With the emphasis on the workpiece quality, the internal defect

\begin{tabular}{|c|c|c|c|c|c|c|}
\hline No. & \multicolumn{3}{|c|}{$\begin{array}{l}\text { The theoretical } \\
\text { output }\end{array}$} & \multicolumn{3}{|c|}{ The actual output } \\
\hline A1 & 0 & 0 & 1 & 0.02132 & 0.01253 & 0.91021 \\
\hline A2 & 0 & 0 & 1 & 0.01023 & 0.02154 & 0.92521 \\
\hline A 3 & 1 & 0 & 0 & 0.89652 & 0.10235 & 0.02365 \\
\hline A4 & 0 & 1 & 0 & 0.02158 & 0.95213 & 0.02157 \\
\hline A5 & 0 & 0 & 1 & 0.01245 & 0.08521 & 0.94587 \\
\hline A6 & 0 & 1 & 0 & 0.01852 & 0.95210 & 0.01658 \\
\hline A7 & 1 & 0 & 0 & 0.89658 & 0.42011 & 0.02856 \\
\hline A8 & 0 & 1 & 0 & 0.02145 & 0.90258 & 0.01589 \\
\hline A9 & 0 & 0 & 1 & 0.12035 & 0.02157 & 0.96324 \\
\hline A10 & 1 & 0 & 0 & 0.95462 & 0.02145 & 0.01856 \\
\hline
\end{tabular}

Table 4: The comparison between the theoretical output and the actual output.

detection technology of the workpiece has been developed. Common internal defect detection technologies of the workpiece include ultrasonic, laser holography, X-ray photography, etc. Industrial CT images are currently the most effective non-destructive testing technology [17], making it easier to identify defects. Defect recognition based on industrial CT images is a simple and efficient method.

Before defect recognition, it is necessary to locate and segment defects in the image. Defect localization can obtain the location information of defects from CT images, including the method of fractal, Gabor wavelet, statistics, etc. Yang et al [18] proposed a localization method based on cubature Kalman smooth filter, which can effectively locate defects. In this study, the block fractal algorithm was selected for image localization, and an industrial CT image was taken as an example. It was found that the algorithm can successfully locate the defect part.

The improved k-means clustering algorithm was selected to segment the image. The experiments showed the accuracy of the algorithm more than $95 \%$, which provided a good foundation for the following defect feature extraction and recognition.

$\mathrm{Hu}$ invariant moment theory was adopted in this study for defect feature extraction. The abstraction of $\mathrm{Hu}$ invariant moment can make it better to extract features. Then, ten feature vectors can be obtained, plus the two shape features and the two gray features equal the total of 14 feature vectors which were used in feature recognition. For feature recognition there exist numerous methods such as artificial neural networks (ANN), support vector machine (SVM), principal component analysis (PCA) and other. In this study, the RBF neural network was selected to recognize the features, and then the weights and the threshold of the neural network were optimized by using the firefly algorithm. The accuracy rate of $97.89 \%$ was obtained in the experiments, which proved the reliability of the defect recognition algorithm in this study.

Industrial CT image is one of the effective methods for non-destructive testing of workpiece. In this study, 
based on the industrial CT image, the image defects were located by the block fractal algorithm, then the improved k-means clustering algorithm was used to segment the defect image, the abstracted $\mathrm{Hu}$ invariant moment algorithm was adopted for feature extraction, and finally the firefly algorithm and the RBF neural network were used for feature recognition.

\section{Acknowledgement}

We completed this paper based on the project of contours extraction from industrial computed tomography images supported by Chongqing Municipal Education Committee.

\section{References}

[1] Alimohamadi H, Ahmadyfard A, Shojaee E. (2010). Defect Detection in Textiles Using Morphological Analysis of Optimal Gabor Wavelet Filter Response. International Conference on Computer and Automation Engineering, IEEE, Bangkok, Thailand, pp. 26-30.

https://doi.org/10.1109/ICCAE.2009.43

[2] Chen Z, Wang C, Hu Z, Xie S. (2010). Dual-tree complex wavelet analysis and its application in defect detection of workpiece for cross wedge rolling. International Conference on Advanced Computer Theory and Engineering, IEEE, Chengdu, China, pp. 861-72. https://doi.org/10.1109/ICACTE.2010.5579227

[3] Sabeenian RS, Paramasivam ME. (2010). Defect detection and identification in textile fabrics using Multi Resolution Combined Statistical and Spatial Frequency Method. Advance Computing Conference, IEEE, Patiala, India, pp. 162-166. https://doi.org/10.1109/IADCC.2010.5423017

[4] Liu BT, Hou DB, Liu BL, Zhao L, Huang PJ, Zhang GX. (2014). Defect detection and identification in eddy current testing using subtractive clustering algorithm combined with RBFNN. Insight - Non-Destructive Testing and Condition Monitoring, 56(7), pp. 375-380(6). https://doi.org/10.1784/insi.2014.56.7.375

[5] Leng Y, Xiao Z, Geng L, Xi J. (2018). Defect detection and classification of galvanized stamping parts based on fully convolution neural network. International Conference on Graphic and Image Processing, pp. 188. https://doi.org/10.4028/www.scientific.net/AMR.6 75.55

[6] Zhang RF. (2013). Study on CT Image Reconstruction Applications in Industry. Advanced Materials Research, pp. 55-58.

https://doi.org/10.4028/www.scientific.net/AMR.6 75.55

[7] Carmignato S. (2012). Accuracy of industrial computed tomography measurements: Experimental results from an international comparison. CIRP Annals - Manufacturing
Technology, 61(1), pp. 491-494.

https://doi.org/10.1016/j.cirp.2012.03.021

[8] Chen L. (2011). The Application and Investigation about Industry CT Scan Technology in the Measure and Design about Complex Box. Applied Mechanics \& Materials, pp. 319-322.

https://doi.org/10.4028/www.scientific.net/AMM.8 6.319

[9] Samarawickrama Y C, Wickramasinghe C D. (2017). Matlab based automated surface defect detection system for ceremic tiles using image processing. Technology and Management, IEEE, 34-39.

https://doi.org/10.1109/NCTM.2017.7872824

[10] Jiang SQ, Luan CB, Man YE, Zhao YY. (2017). Application of Industry CT in Large Complicated Casing Inspection. Nondestructive Testing, 39(2), pp. $18-21$. https://doi.org/10.1109/NSSMIC.2011.6154616

[11] Chang M, Xiao Y, Chen Z, Li L. (2011). Preliminary study of rotary motion blurs in a novel industry CT imaging system. Nuclear Science Symposium and Medical Imaging Conference. IEEE, Valencia, Spain, pp. 1358-1361. https://doi.org/10.1109/NSSMIC.2011.6154616

[12] Zabler S, Fella C, Dietrich A, Nachtrab F, Salamon M, Voland V, Ebensperger T, Oeckl S, Hanke R, Uhlmann N. (2012). High-resolution and highspeed CT in industry and research. SPIE Optical Engineering + Applications, San Diego, California, United States, pp. 850617. https://doi.org/10.1117/12.964588

[13] Mandelbrot BB, Wheeler JA. (1998). The Fractal Geometry of Nature. American Journal of Physics, 51(4), pp. 468 p. https://doi.org/10.1119/1.13295

[14] Normand MD, Peleg M. (1988). Evaluation of the 'blanket' algorithm for ruggedness assessment. Powder Technology, 54(4), pp. 255-259. https://doi.org/10.1016/0032-5910(88)80055-X

[15] Tang YH, Gong A, Wang C. (2012). Automatic Generation Algorithms Based on Optimization Initial Population. Computer \& Modernization, 562(1), pp. 131-147.

[16] Yan BJ, Zheng L, Wang KY. (2001). Fast TargetDetecting Algorithm Based on Invariant Moment. Infrared Technology, 23(6), pp. 8-12.

[17] Xie L, Huang R, Gu N, Cao Z. (2014). A novel defect detection and identification method in optical inspection. Neural Computing \& Applications, 24(7-8), pp. 1953-1962. https://doi.org/10.1007/s00521-013-1442-7

[18] Yang L, Li H, Zhou F, Jin P. (2015). The Pipeline Defect Location Technology Based on Cubature Kalman Smooth Filter. Chinese Journal of Sensors \& Actuators, 28(4), pp. 591-597. https://doi.org/10.3969/j.issn.10041699.2015.04.023 